\title{
Wireless Underground Sensor Networks Channel Using Energy Efficient Clustered Communication
}

\author{
R. Kanthavel ${ }^{1, *}$ and R. Dhaya ${ }^{2}$ \\ ${ }^{1}$ Department of Computer Engineering, College of Computer Science, King Khalid University, Abha, Saudi Arabia \\ ${ }^{2}$ Department of Computer Science, College of Arts and Science-Sarat Abidha, King Khalid University, Abha, Saudi Arabia \\ *Corresponding Author: R. Kanthavel. Email: kanthavel2005@gmail.com \\ Received: 25 April 2021; Accepted: 02 June 2021
}

\begin{abstract}
Wireless Underground Sensor Networks (WUSNs) refer to a group of nodes working underneath the earth plane that has been predicted to render concurrent observation capability in the hostile subversive and underwater surroundings. The accurate monitoring in places like underground earth, water, lubricates so on called non-conventional media need high accuracy of tiny sized sensors with antennas at a similar size. Therefore, an investigation is needed to study the opportunities and drawbacks of utilizing WUSNs without compromising the effectiveness of real-time monitoring procedures. With this, the major confrontation is to institute a trustworthy underground communication regardless of the complex environment that has the key difficulties like elevated path loss, and miniature communication range when piercing through the soil, sand, water, and oil surfaces. On the other hand, aside from on or after elevated pathway failure, it acknowledges continuously varying channel deeds owing to the underground communication. In non-conventional media, dealing with the concerns for scheming cluster-based cooperative communication protocols for sensors have been a tough task to improve the network lifetime and its energy effectiveness. The paper intends to concentrate on these problems to put forward a fresh cooperative communication channel model bearing in mind, the propagation belongings of terahertz EM waves in the underground sensor network. Besides, the work also focuses on the creation of energy-efficient Clustered Cooperative communication protocol (CCNU) for underground sensor network to yield better reliability and effective utilization of spectrum efficiency by obtaining better lifetime, throughput, packet loss, successful packet delivery, and energy utilization. The outcome of this proposal would yield an outcome in the form of better communication channel modeling for monitoring, intrusion detection, exploration, and sensing.
\end{abstract}

Keywords: Underground wireless sensor networks; cooperative communication; channel modeling; packet delivery

This work is licensed under a Creative Commons Attribution 4.0 International License, which permits unrestricted use, distribution, and reproduction in any medium, provided the original work is properly cited. 


\section{Introduction}

Cooperative communications allow well-organized use of communication resources, through permitting nodes or terminals in a communication network to work together through every additional information communication. Cooperative communications is also capable of considerable progress the system variety of competence and performance. Furthermore, it is based on transmitting nodes that have materialized as a promising mechanism for the quality parameters namely spectral and power competence, network exposure, and to decrease outage possibility [1].

The classification in two divisions namely Single and multi hoping are playing significant roles in underwater cooperative communication and out of which multi-hop communication method has been the best choice for transferring information between source and destination by carefully taking the parameters called restricted bandwidth, multipath fading, restricted battery, incomplete information capability and propagation delay [2]. Wireless communication networks used Underwater comprise sensors and independent underwater vehicles that network, organize and split data using every additional to take out intelligence and check utilities. The submarine makes use of a dedicated telephone network to converse underwater utilizing functioning like an Amplitude Modulation-based wireless signal, except for that it broadcasts and obtains sound waves as a replacement for the transmission and reception radio waves. Alike to land communication method, underwater telephone networks make use of microphones and acoustic speakers [3].

The research problems identified are first while deploying the UWSNs in the hostile environments with the crack thickness under $100 \mathrm{~mm}$. The sensor nodes dimension should be in the millimeter magnitude in the operating frequency of Gigahertz or Terahertz range. Secondly, path loss and lack of transmission radius happen in non-invasive transmission medium like water and liquid matters a radio signal of $\mathrm{GHz}$ frequency range and $\mathrm{THz}$ range [4] will incident rigorous intermediate assimilation. Thirdly, while encountering severe energy problems in UWSNs because of its deployment regions in complex environments as the tiny sensors merely consent to incredibly tiny storage of power using exceptionally restricted power delivery. Fourthly owing to the unsuitable renewable energy-producing methods make the need for a cluster-based cooperative communication network to add more reliability in quality of service. The aforementioned precise of WUSN and the demanding issues they pretense demand to enlarge a novel power-effective signal propagation forms and methods [5].

The objectives of this paper are to analyze the power consumption for the projected cooperative communication method in Underground WSNs cooperative transmission environments using clusters as its main part. To model power proficient cluster-based communication protocols for Underground WSNs are: To examine the realistic communication expanse among cooperative communications sensors for various underground matters. To put forward a new cooperative communication model taking into account the propagation possessions of EM waves in the difficult environments of the subversive. The organization of this research finding molds into four main sections namely analysis of existing schemes in cooperative communication for underground sensor networks, Efficient Wireless Underground Sensor Networks channel modeling (WUSNs) using Energy-efficient Clustered Cooperative Communication, and experimental results and outcome.

\section{Literature Review}

This section renders the various literature studies about the energy efficient Clustered Cooperative communication models for underground surveillance and previous underground sensor network Underground monitoring researches. Underground surveillance is a very complex process because of the involvement of plenty of resources that needs to be observed much information as possible at each stage of the process of sensing, computation and communication in the Underground wireless sensor nodes [6]. 
The researchers have been in search of various modern methods to answer by taking out real time data from the hostile underground environments using cooperative communication techniques to increase life time and energy in Wireless Underground Sensor networks efficiently. The literature survey has been done in three categories namely the current status of WUSNs and its merits and restrictions in terms of hostile underground environment, the utilization of cluster based cooperative communication techniques in WSN, the existing problems at the receiver side in spite of the heavy path loss considering milli-meter wave transmission and the wireless channel model [7].

The main challenge for comprehending WUSNs is assessing the wireless channel by taking its connected nodes. The wireless channel propagation mechanisms in the form of reflection, diffraction and refraction have an effect on the wireless transmission making the receiver network hard to envisage the received signal. The chief tools for analyzing Channel modeling and simulation cost effectively [8]. WUSNs have been a new-fangled model but with only a small number of researches that deals with the problems regarding underground communication channel modeling. The mechanism on learning the belongings of the wireless underground channel focal point on the categorization based on above and under the earth at the time of detaining the possessions of underground environments with respect to frequency and other parameters and the deployment of sensors underground [9]. The propagation characteristics have also been investigated through simulation with the results for two adjacent nodes. Hence, modeling a wireless channel is a challenging task in underground sensor networks. Liu et al. learnt the complex ways of monitoring the position of underground matters that results from various researches connected to the examination of acoustic wave's possessions in different media. Their analysis also includes various underground environments and their reflection properties. In $\mathrm{Xu}$ et al. explained underground monitoring studies related with oilfield associated gas process using WUSNs in cooperative communication environments. Mazzini et al. deliberated the chance of taking an automatic mechanism for concrete mapping sensors in aggressive surroundings.

Zhi et al. intended and executed a model on Magnetic Induction (MI) based communications for WUSN, but unsuccessful to put into operation on cooperative communication system to decrease the system power. Also, Zhi et al. presented an MI wave guide system to decrease the far above the ground path failure owing to the pursued way of the customary EM signal. Akyildiz et al. devised an examination of the existing sensor networks. They were also unsuccessful to acquire the energy necessities of various sensor networks in terms of restricted utilization in underground communication systems. They also executed cooperative communication energy efficiently by means of calculating Bit Error Rate in cluster based network examination. However they tend to fail to consider the cooperative communication that applied just in support of predictable means that are not suitable for unpredictable medium. Li et al. deliberated a cooperative transmission system for arbitrary distribution of sensor network that uses RF for communication. They have also analyzed performance analysis by formulating approaches to reduce the whole energy. But, they did not reflect on other unpredicted situation in their effort in the environment where heavy path loss occurs. Kumar et al. produced an energy efficient communication model to analyze its efficiency for three different non-predictable media in order to reduce the energy utilization more. Foran et al. underwent a Very near gave a study on the use of radio waves in military applications. Isler et al. provided a solution for sampling based Sensor-Network deployment.

Sailhan et al. proposed Monitoring methods of Wireless Mesh Network which includes design, implementation and experiments. Gungor et al. studied the disputes, design values, and technological advancements of manufacturing WSNs. Kandris et al. discussed the major groups of applications of WSNs are recognized, and attribute cases that includes the WUSNs complex environment. Tam et al. tackled the restrictions of positioning relay nodes to minimize communication loss in a load matching restriction through suggesting two estimation algorithms to enhance the outcome got. Sambo et al. evaluated WUSN, concentrated dimensions on real sensors by way of two special duos of transceivers 
under complex environments. From the above studies, it is understood that though there have been many researches on WSUNs for analyzing the acquisition of real time data. But in almost all results it is apparently learned that the challenges like efficient energy utilization and extreme path loss have not been considered.

The school of thought from the literature survey is that the proposal needed here must possess the purpose of the utmost communication distance with the consideration of the entire path loss and the operational frequency in the varied underground environments. Also there is a much need to establish the prolongation of a feasible broadcast window of cluster based cooperative communication development in this detailed category of WUSNs.

\section{Need for Cooperative Communication for Underground Surveillance Network}

To transfer signals in underground communication systems, electromagnetic and sonar waves have been in use either individually or together as underwater communication which is not easy owing to aspects that include multi-path propagation, time differences in channel duration, little accessible bandwidth, and rigorous signal reduction, particularly above extensive assortment [10]. The cooperative communication model is shown in Fig. 1. In the proposed model, Tx, Rx, TR, RR, CH represent Transmitter node, Receiver Node, Transmitter Relay Node, Receiver Relay Node, and Cluster Head Node respectively. The communication flow happens between TX-TR-CH-RR-RX nodes serially so as to have the connectivity in order for the underwater environment. The frequency range between 3 and $30 \mathrm{kHz}$ called very low that is capable to go through seawater to hardly any tens of meters that enable the communication for an essential mechanism of electronic infrastructure system like sender node, communications medium, receiver node, and noise. The transmission and reception of sound waves and digital signals using analog and digital are difficult in underground environments than air as water has high permittivity and conductivity [11]. On the other hand, the high attenuation of plane waves contrasts with air concerning frequency. Moreover, Wi-Fi does not function well in underwater instead it is passed on via wires. In addition to that radio waves do not have the potential to pass through excessively distant and microwaves are the better option as seeing water does not conduct electricity well at the underground frequencies [12]. But for underwater frequencies, the reception above ground desires to be wholly shielded as of the adjoining water [13]. For the above reasons, the most significant issue in underground wireless cooperative communications system design needs to have been improved by utilizing spectral efficiency and association consistency amid multipath fading and interference that happen in the underground environment [14]. In the proposed communication model, the term cooperative mechanism comes in to act for forwarding the data from the source node to destination node through the cooperative nodes namely TR, $\mathrm{CH}$ and RR. The specialty of this model is to ensure the reliability as the nodes have been deputed serially.

Energy Efficient Clustered Cooperative Communication Based Channel Modeling For WUSNs: Energy-efficient Clustered Cooperative Communication based Channel Modeling protocol is proposed. The overall WUSNs environment is shown in Fig. 2. This model has two layers namely Layers 1 and 2. Every Cluster head has been interconnected with each other which can contact the destination node in the communication Medium. The nodes that are in every layer can send the collected data to their respective cluster head before each cluster gets connected with other clusters in the communication model. In such a way that the acquired data from all clusters finally forwards towards the destination node. The usage of layers in the communication model is to prefer the superior cluster head for making the communication reliable. The significance of the cluster head selection in the proposed model is to create firm connectivity among its nodes so as to have the full control mechanism over the data transfer. 


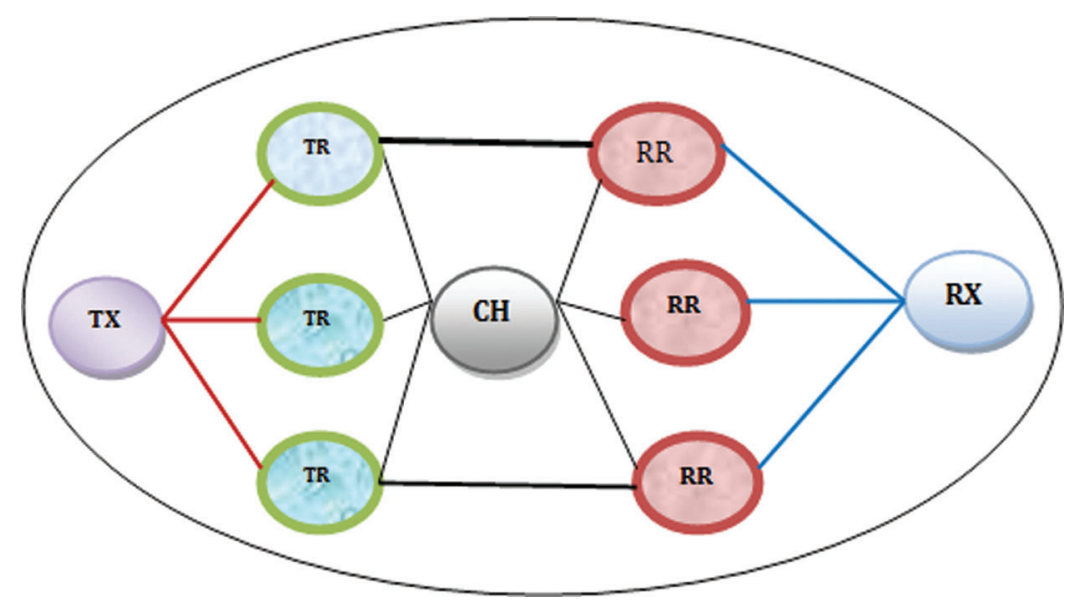

Figure 1: Cooperative communication model

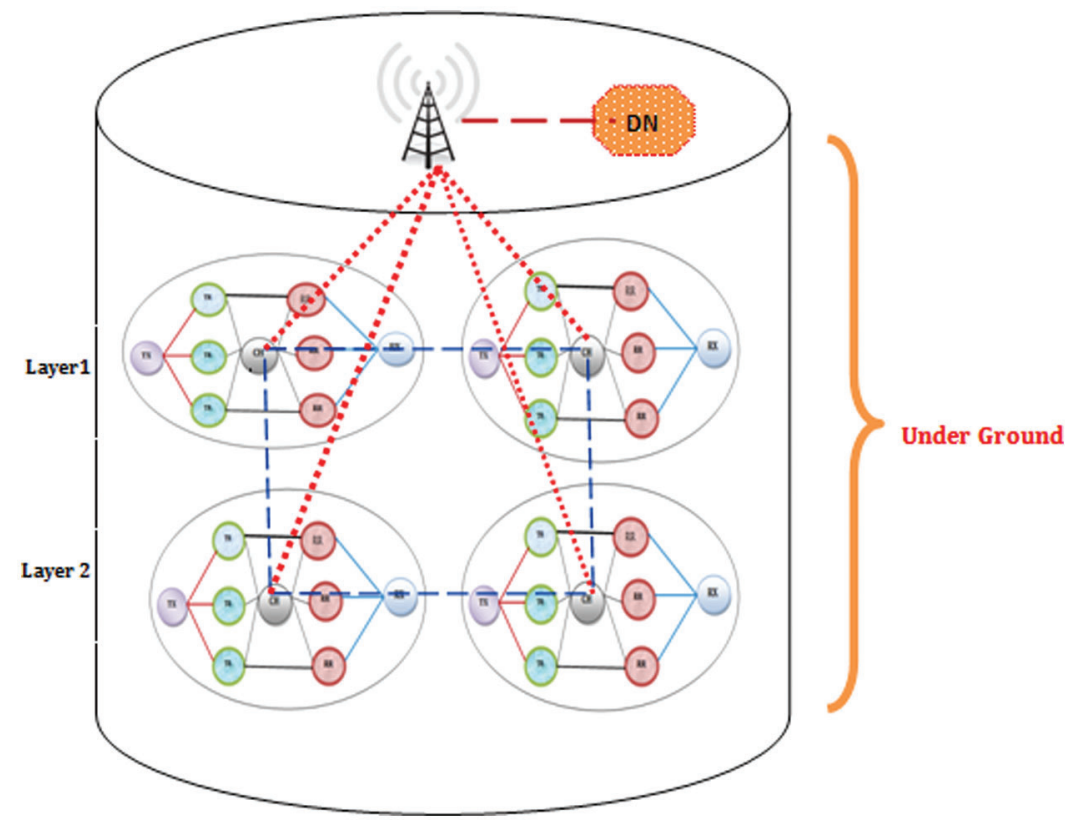

Figure 2: Proposed underwater cooperative communication model

Cooperative communication is meant here in the underwater environment for utilizing the relay nodes in between source and destination for the effective and efficient communication. The Destination node in the proposed communication model has the responsibility to receive the data from its cluster heads in order by the cooperative nodes as the effective set up. The highlights of the proposed model are the superior selection of layers and cluster head for checking the quality links in order to ensure cooperative nodes data qualitatively.

\section{Experimental Results and Outcomes}

The testing of this simulation of the Energy-efficient Clustered Cooperative Communication is done based Channel Modeling protocol. The first part of the simulation is to produce the WUSNs simulation 
channel model and the channel is assumed to have been accommodated with 4 clusters on which there have been cluster head and cluster nodes. The nodes inside every cluster gather information within its range of underground and the same is forwarded to the control node [15]. All cluster heads have been connected logically and physically to communicate with each other in terms of exchanging the relevant information quickly for further transmission to keep up the reliability and avoiding delay spread [16]. The proposed WUSN communication flow is illustrated in Fig. 3. It is assumed that the TxR is responsible for data transmission after sensing and $\mathrm{Rx}$ does the work of reciprocal of the transmitter in terms of data reception. TxR has the potential to relay the information sent by the transmitter and $\mathrm{RxR}$ does the reception work of receiver node data. The prime role of this proposed cooperative communication surveillance model is the functional property of Cluster Head which is doing a major role in connecting the layer to the control node. In addition to that, the cluster node has the privilege not to forward the data, if the quality of information is up to the marks by lack of clarity [17]. The quality link assessment is needed here while selecting the layer for making the communication continuously without any interruption since the model incorporates many interconnected nodes. The full data transfer is done successfully by choosing either layer 1 or 2 for the better cluster head in order to ensure the data arrival as the cluster head holds the entire control over the communication set up.

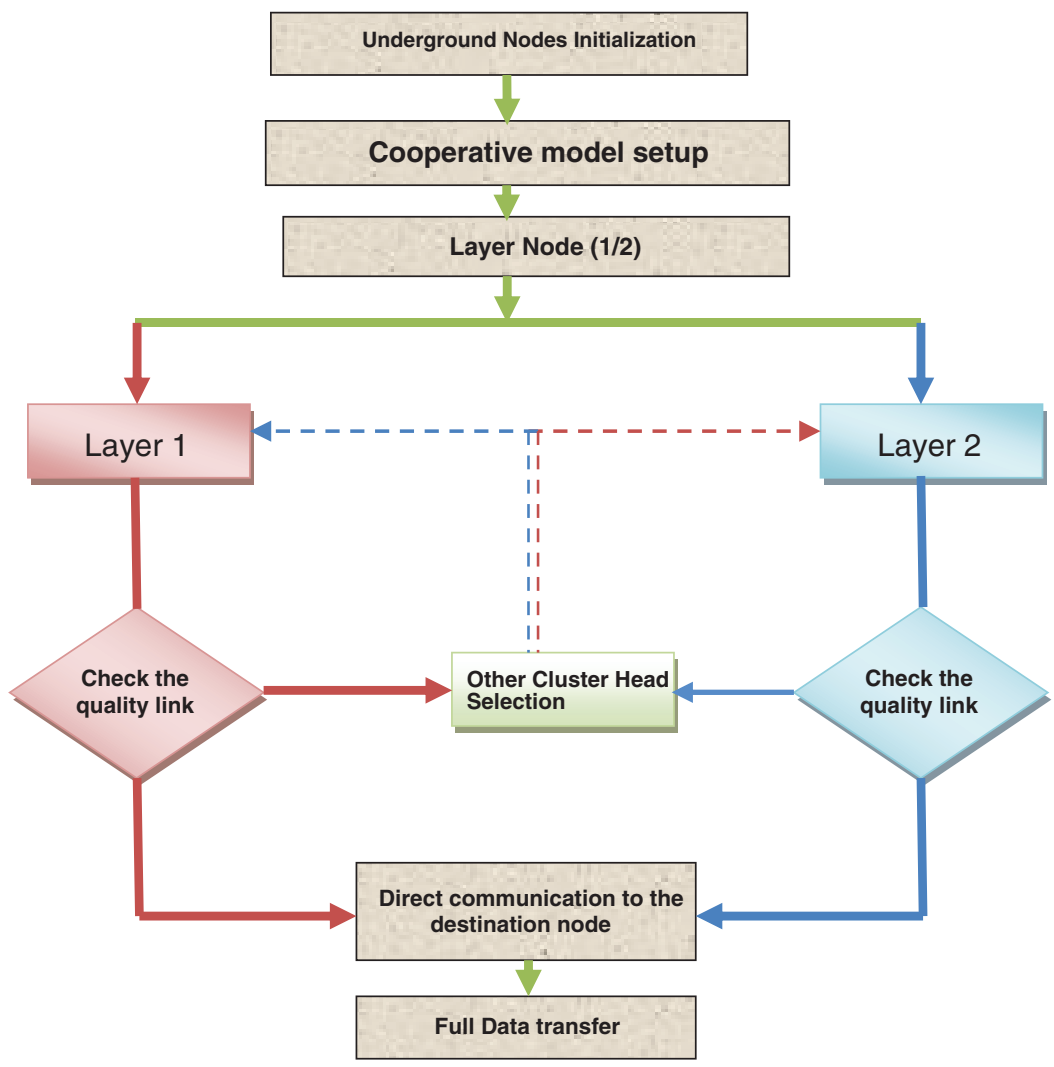

Figure 3: Proposed WUSN communication flow

\section{Results \& Discussion}

This paper uses Network Simulator 2 to investigate the performance with network lifetime and throughput. The environment is set in Tab. 1 as the parameters to experiment under. The network layer bifurcation is based on the area in which the surveillance needs to be undertaken. The communication 
radius of all the nodes is $500 \mathrm{~m}$ and it is assumed that the nodes are uniformly distributed and timesynchronized. The need of network scale is to design the communication model as a coverage area limitation in which the nodes can communicate among themselves as mentioned in the table. The number of nodes used here in the proposed communication model is 100 with two layers. Further the addition of nodes in terms of scalability depends upon the area and communication radius. The need of measuring noise power here is to calculate the quality of receiving data and it is related with the bandwidth proportionally.

Table 1: Factor setting values for simulation

\begin{tabular}{ll}
\hline Network scale & $5 \times 5(\mathrm{~km})$ \\
Number of nodes & 100 with 2 layers \\
Communication radius & $500 \mathrm{~m}$ \\
Energy allotted per node & $5 \mathrm{~J}$ \\
Data length & $\mathrm{LD}=1000 \mathrm{bits}$ \\
Received power of nodes & $R P_{n}=0.0025$ \\
Noise power & $\mathrm{NP}_{\mathrm{n}}=0.0006$ \\
Boost up energy of relay nodes & $B E_{R}=0.003$ \\
frequency & $\mathrm{F}=5 \mathrm{KHz}$ \\
\hline
\end{tabular}

The signal strength can be calculated with the following equations.

$$
\begin{aligned}
& A_{T x R x}=T_{S} C_{T x R}+N_{T x R} \\
& A_{T x R}=T_{S} C_{T x R}+N_{T x R} \\
& A_{R x R}=T_{S} C_{R x R}+N_{R x R}
\end{aligned}
$$

where

- $\mathrm{A}_{\mathrm{TxRx}}$ defines signal power from source and receiver node;

- $\mathrm{A}_{\mathrm{TxR}}$ refers source and source Relay node signal strength ;

- $A_{R \times R}$ denotes signal strength from Receiver node and Receiver Relay node;

- $\mathrm{T}_{\mathrm{S}}$ means initial signal strength of the transmitter node;

- $\mathrm{C}_{\mathrm{TxR} x}$ indicates Channel fading value of the Transmitter node and Receiver node;

- $\mathrm{C}_{\mathrm{TxR}}$ represents Channel fading value of the Transmitter node and Relay node;

- $\mathrm{C}_{\mathrm{RxR}}$ means Channel fading value of the Relay node and Receiver node;

\section{Simulation Analysis}

The proposed energy-efficient Clustered Cooperative communication model is experimented to measure out the performance with the parameters that include lifetime, throughput, packet loss, successful packet delivery, and energy utilization of the network. The lifetime of the network is measured through the time spend for the beginning to end energy sustainability. Throughput can be calculated the successful reception of packets per stimulated time. 
The calculation of the Packet Loss is done by seeing the unsuccessful reception of data packets [18]. Successful packet delivery is nothing but the calculation between successful packet reception and packet loss in terms of the packet sent [19]. Energy utilization is the measure of adding all transactions between source and destination in terms of energy. 400 nodes have been employed with an area of $5 \mathrm{~km} \times 5 \mathrm{~km}$ in which all the nodes are given initial energy as $5 \mathrm{~J}$ utilizing static communication range with a fixed transmission range of $500 \mathrm{~m}$. 1000 bits is fixed as the length of the data to be used for data transactions. The above settings have been made in the simulation environment for the underground wireless cooperative communication model.

Fig. 4 shows the network length evaluation for the projected CCNU as well as the existing Energy Efficient Depth Based Routing protocol (EEDBR) by plotting the number of dead nodes versus time. It is understood that the lifetime of the proposed network has been improved significantly than the EEDBR which is due to the nodes' intelligent function in terms of energy utilization as the clustered concept has been inbuilt in the layered network.

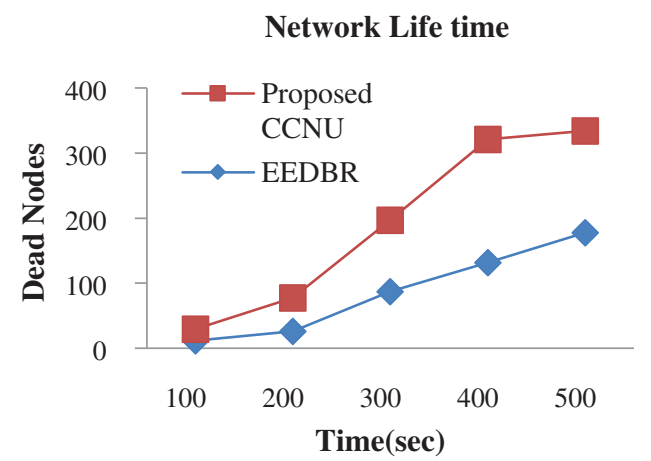

Figure 4: Network life time

The energy utilization graph has been plotted in Fig. 5, which shows the plot drawn between the time and energy utilization for the proposed CCNU and existing EEDBR. From the energy utilization graph, it is inferred that the proposed protocol has an edge over the existing protocol by the way of complete energy utilization successfully to enhance the network qualitatively.

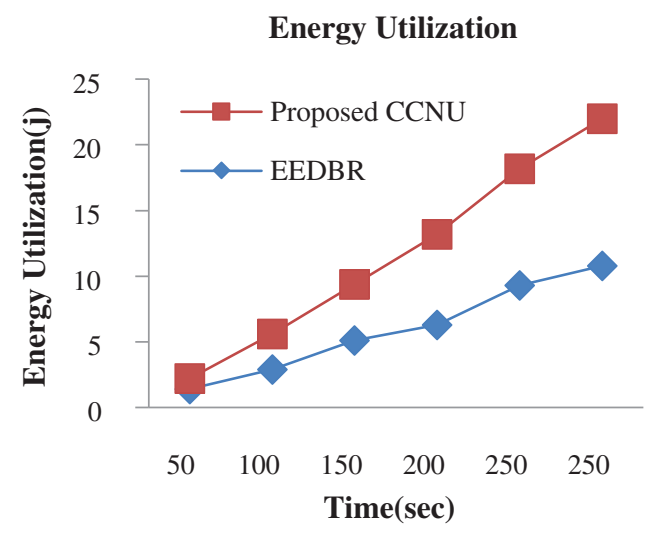

Figure 5: Energy utilization 
IASC, 2022, vol.31, no.1

The throughput calculation is done by means of measuring the time vs. packet received shown in Fig. 6. The proposed protocol has been so attractive in attaining better throughput for different iterations without compromising the lagging of time.

The packet loss and Successful packet delivery have been compared with time vs. packet loss and time versus packet delivery ratio respectively which are shown in Figs. 7 and 8 . The reason behind the successful packet delivery is owing to the proper connectivity that is provided by the proposed CCNU through it's layered cum clustered architecture and the packet loss of the proposed CCNU is not at all affected by the matter of transaction between transmitter and receiver.

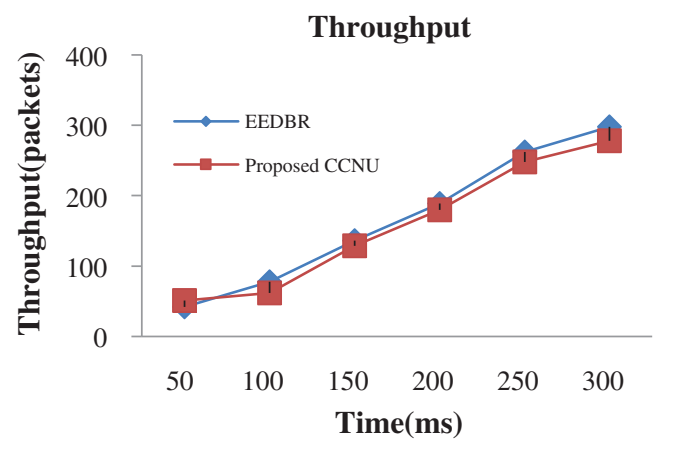

Figure 6: Throughput



Figure 7: Packet loss

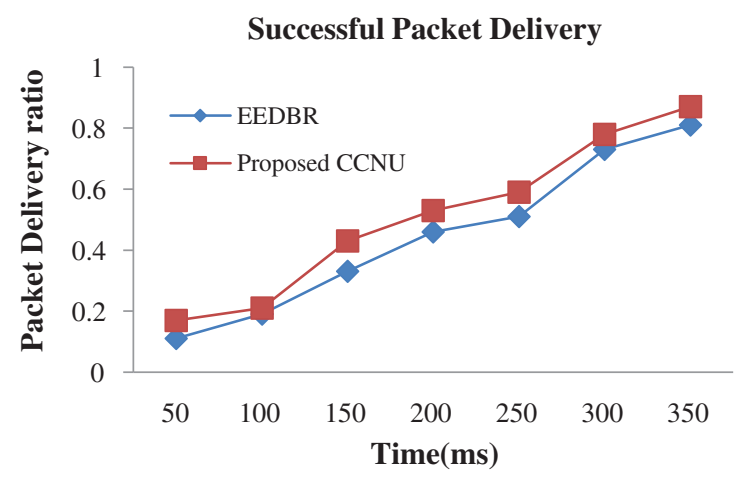

Figure 8: Successful packet delivery 


\section{Summary and Discussion}

The vital suggestions are as follows: The proposed work examined WUSN's communication methods for facilitating well-organized cooperative communication in difficult underground surroundings. This projected model is allowing for the propagation characteristics of EM waves in the hostile underground situation to have been much needed. Furthermore, the assessment of the possible communication distances among nodes and performance assessment concerning lifetime, throughput, packet loss, successful packet delivery, and energy utilization for different underground hostile environments needs to be done to make the network reliable. On the other hand, it is very much essential to elaborate on the essentials and boundaries of using the EM waves with minute range for underground communication. As a result of the proposal, the effective and efficient utilization WUSNs along with the cluster-based cooperative communication (CCNU) model could be well investigated in complex underground environments that will be useful in applications like monitoring, intrusion detection, and exploration and sense than the existing EEDBR Protocol. The requirement of efficient utilization of spectrum efficiency is achieved in the proposed model by means of cluster head selection approach of its layer through the minimum utilization of energy but not compromising the quality. The limitations in the existing model that includes lack of reliability because of not considering hostile environment as well as not taking more QoS parameters which have been overcome as limitations in the proposed model.

\section{Conclusions}

This paper presented the Efficient Wireless Underground Sensor Networks (WUSNs) channel modeling using Energy-efficient Clustered Cooperative Communication. The intention of concentrating on these problems to put forward a new cooperative communication guidance model in underground sensor network has been experimented in simulation. Also, the energy-efficient Clustered Cooperative communication protocol (CCNU) has been applied to render enhanced reliability and efficient use of spectrum than the existing EEDBR Protocol using lifetime, throughput, packet loss, successful packet delivery, and energy utilization. From the proposed work it has been concluded that the cluster cooperative communication-based sensor network provides superior communication channel modeling for observing interruption discovery, and investigation and sensing in multi hop environment. In the future, the work will be having a scope on the elaboration of introducing a model for the communication network using cooperative communication by considering energy efficiency as a paramount tool in cluster-based hierarchical architecture.

Acknowledgement: This research was supported by King Khalid University Researchers Supporting Project number (RGP/108/42), King Khalid University, Abha, Saudi Arabia.

Funding Statement: The authors would like to thank King Khalid University, Abha, Saudi Arabia for funding this work through Small Research Project under Grant Number RGP/108/42.

Conflicts of Interest: The authors declare that they have no conflicts of interest to report regarding the present study.

\section{References}

[1] I. F. Akyildiz, Z. Sun and M. C. Vuran, "Signal propagation techniques for wireless underground communication networks," Physical Communication, vol. 2, no. 3, pp. 167-183, 2009.

[2] M. C. Vuran and I. F. Akyildiz, "Channel model and analysis for wireless underground sensor networks in soil medium," Physical Communication, vol. 3, no. 4, pp. 245-254, 2010. 
[3] F. Mazzini, D. Kettler, J. Guerrero and S. Dubowsky, "Tactile robotic mapping of unknown surfaces, with application to oil wells," IEEE Transactions on Instrumentation and Measurement, vol. 60, no. 2, pp. 420429, 2011.

[4] M. Peng, Y. Li, T. Q. S. Quek and C. Wang, "Device-to-device underlaid cellular networks under rician fading channels," IEEE Transactions on Wireless Communications, vol. 13, no. 8, pp. 4247-4259, 2014.

[5] M. Kyro, K. Haneda, J. Simola, K. I. Takizawa, H. Hagiwara et al., "Statistical channel models for $60 \mathrm{GHz}$ radio propagation in hospital environments," IEEE Transactions on Antennas and Propagation, vol. 60, no. 3, pp. 1569-1577, 2012.

[6] P. Allen and P. Michelman, "Acquisition and interpretation of 3-D sensor data from touch," IEEE Transactions on Robotics and Automation, vol. 6, no. 4, pp. 397-404, 1990.

[7] Z. Sun and I. F. Akyildiz, "Connectivity in wireless underground sensor networks," in 7th Annual IEEE Communications Society Conf. on Sensor, Mesh and Ad-hoc Communications and Networks, Boston, MA, USA, pp. 1-9, 2010.

[8] Z. Sun and I. F. Akyildiz, "Deployment algorithms for wireless underground sensor networks using magnetic induction," in IEEE Global Telecommunications Conf. GLOBECOM, Miami, FL, USA, pp. 1-5, 2010.

[9] I. F. Akyildiz, W. Su, Y. Sankara Subramaniam and E. Cayirci, “A survey on sensor networks," IEEE, vol. 40, no. 8, pp. 102-114, 2002.

[10] Z. Zhou, S. Zhou, S. Cui and J. Cui, "Energy-efficient cooperative communication in a clustered wireless sensor network," IEEE Transactions on Vehicular Technology, vol. 57, no. 6, pp. 3618-3628, 2008.

[11] B. Li, W. Wang, H. Li, Y. Zhang and H. Liu, "Performance analysis and optimization for energy efficient cooperative transmission in random wireless sensor network," in IEEE Int. Conf. on Communications, Budapest, Hungary, pp. 1635-1639, 2013.

[12] V. Isler, S. Kannan and K. Daniilidis, "Sampling based sensor-network deployment," in IEEE-RSJ Int. Conf. on Intelligent Robots and Systems, vol. 2, pp. 1780-1785, Sendai, Japan, 2004.

[13] V. Kumar, R. Bhusari, S. B. Dhok, A. Prakash, R. Tripathi et al., "Design of magnetic induction based energyefficient wsns for nonconventional media using multilayer transmitter-enabled novel energy model," IEEE Systems Journal, vol. 13, no. 2, pp. 1285-1296, 2019.

[14] F. Sailhan, L. Fallon, K. Quinn, P. Farrell, S. Collins et al., "Wireless mesh network monitoring: Design, implementation and experiments," in IEEE Globecom Workshops, Washington DC, USA, pp. 1-6, 2007.

[15] R. A. Foran, T. B. Welch and M. J. Walker, "Very near ground radio frequency propagation measurements and analysis for military applications," in IEEE Military Communications Conf. Proc., Atlantic City, NJ, USA, vol. 1, pp. 336-340, 1999.

[16] V. C. Gungor and G. P. Hancke, "Industrial wireless sensor networks: Challenges, design principles, and technical approaches," IEEE Transactions on Industrial Electronics, vol. 56, no. 10, pp. 4258-4265, 2009.

[17] D. Kandris, C. Nakas, D. Vomvas and G. Koulouras, "Applications of wireless sensor networks: An up-to-date survey," Applied System Innovations, vol. 3, no. 1, pp. 14, 2020.

[18] N. T. Tam, D. A. Dung, T. H. Hung, H. T. Binh and S. Yu, "Exploiting relay nodes for maximizing wireless underground sensor network lifetime," Applied Intelligence, vol. 50, pp. 4568-4585, 2020.

[19] D. Wohwe Sambo, A. Forster, B. O. Yenke, I. Sarr, B. Gueye et al., "Wireless underground sensor networks path loss model for precision agriculture," IEEE Sensors Journal, vol. 20, no. 10, pp. 5298-5313, 2020. 